\title{
Digital Mental Health
}

\author{
Nikolaos Boumparis, MSc ${ }^{1}$ \\ Mieke H. J. Schulte, $P h D^{1}$ \\ Heleen Riper, $P h D^{1,2, *}$
}

\author{
Address \\ ${ }^{*}, 1$ Department of Clinical, Neuro- and Developmental Psychology, Amsterdam \\ Public Health Research Institute, Vrije Universiteit Amsterdam, Van der \\ Boechorststraat 7, 1081 BT, Amsterdam, The Netherlands \\ Email: h.riper@vu.nl \\ ${ }^{2}$ Department of Research and Innovation, GGZ inGeest Specialized Mental Health \\ Care, Amsterdam, The Netherlands
}

Published online: 26 November 2019

(C) The Author(s) 2019

Nikolaos Boumparis and Mieke H.J. Schulte contributed equally to this work.

This article is part of the Topical Collection on Technology and its Impact on Mental Health Care

Keywords Substance use $\cdot$ Treatment $\cdot$ Intervention $\cdot$ Digital $\cdot$ Internet $\cdot$ Web-based

\begin{abstract}
Purpose Only about $20 \%$ of people suffering from substance use disorders access available treatments due to various obstacles; digital interventions could potentially overcome some of these. Meta-analyses suggest the strongest evidence for interventions targeting alcohol use reduction, followed by cannabis and illicit substances. However, most randomized controlled trials (RCTs) used unguided standalone interventions compared to non-active controls, with limited follow-up periods and disregarded comorbidity. This review examines the literature published over the last three years (2016-2019), with a focus on recent RCTs and whether they addressed some of these gaps.

Recent findings Except for digital interventions targeting alcohol use, the number of RCTs in the last three years is limited. Although there is considerable heterogeneity between the studies, most of them applied unguided add-on interventions compared to active control groups, and a limited number investigated guided interventions. In addition, there is a need for longer follow-up periods, active rather than non-active control groups, outcome standardization, and increased focus on comorbidity.

Summary Although the number of studies using guided add-on or blended interventions compared to active controls has increased, future studies should consider our identified gaps and suggestions to further strengthen the evidence of digital interventions for reducing the use of alcohol and other substances.
\end{abstract}




\section{Introduction}

The use of alcohol and other substances is a major public health problem associated with detrimental physical, psychological, and social consequences. Global prevalence of substance use disorders (SUDs) has been estimated at around 2.4\% (about 314 million individuals) $[1,2]$. According to the World Health Organization, SUDs globally account for approximately 21 million disability-adjusted life years lost yearly [3], indicating a significant societal and economic burden. SUDs are characterized by high rates of psychiatric comorbidity, such as anxiety disorders, mood disorders, and a higher likelihood for suicidal behavior [4-6].

A wide variety of psychosocial and behavioral treatments are available, ranging from brief interventions to cognitive behavioral therapy (CBT), contingency management (CM), motivational interviewing (MI), 12-step facilitation therapy (TSF), and the community reinforcement approach (CRA). When delivered face-to-face, these treatments have been shown to be effective in reducing substance use with significant moderate to small effect sizes [7-10] when compared to non-active or treatment control conditions, respectively.

However, depending on the used substance, only approximately 7.5 to $20 \%$ of people with an SUD make use of any treatment services [11-13], which has been explained by perceived stigma, unavailable treatment services, forced abstinence as a goal, or time conflicts between participating and other obligations [14-16]. Digital interventions could contribute to partly overcoming these obstacles due to their potential to lower the threshold to access treatment, increased perceived anonymity when following treatment at a distance, and greater availability of treatment independent of time and place [17]. Meta-analyses suggest that digital interventions are effective in reducing substance use when compared to non-intervening controls and may have results similar to face-to-face interventions. The strongest evidence exists for interventions targeting alcohol use [18•], followed by cannabis [19•] and illicit substances [20 $]$. Based on the literature, it appears that for alcohol and cannabis, most randomized controlled trials (RCTs) investigated unguided standalone interventions compared to non-active controls. For psychostimulants, the picture is somewhat different; two RCTs studied unguided standalone online interventions and two studied online interventions that were "added on" to treatment-as-usual interventions (TAU). Regarding opioids, only guided add-on interventions that were compared with active conditions had been published in the past. Additionally, it needs to be emphasized that the number of RCTs applying digital interventions targeting psychostimulant and opioid use is scarce $[18 \bullet, 19 \bullet, 20 \bullet]$. Furthermore, with respect to all substances, there is a need for longer follow-up periods, outcome standardization, comparisons with active rather than non-active conditions, and an increased focus on comorbid conditions.

This review will examine the literature published over the last three years (2016-2019), with a focus on RCTs and on whether these new studies targeted some of the above-mentioned gaps. Standardized interventions delivered via the internet (internetbased) and standardized interventions delivered via a software program on a computer (computer-based) were included. The term "digital interventions" is used to refer to both internet- and computer-based interventions. RCTs targeting exclusively university students were excluded as they examine student drinking patterns which mainly entail "binge drinking." Studies making use of experimental approaches, such as bias modification and cognitive skills training, were also excluded as these interventions differ substantially from behavioral ones. Given that studies define high drinking levels differently and use varying terms to define problematic users (e.g., problem drinkers, risky drinkers, heavy drinkers), the term "problem drinkers" was used while describing the studies. Included studies are summarized in Table 1.

\section{Reduction of alcohol use}

\section{Personalized normative feedback}

Personalized normative feedback (PNF) interventions provide tailored feedback delivered via self-report tools measuring substance use. The feedback is 


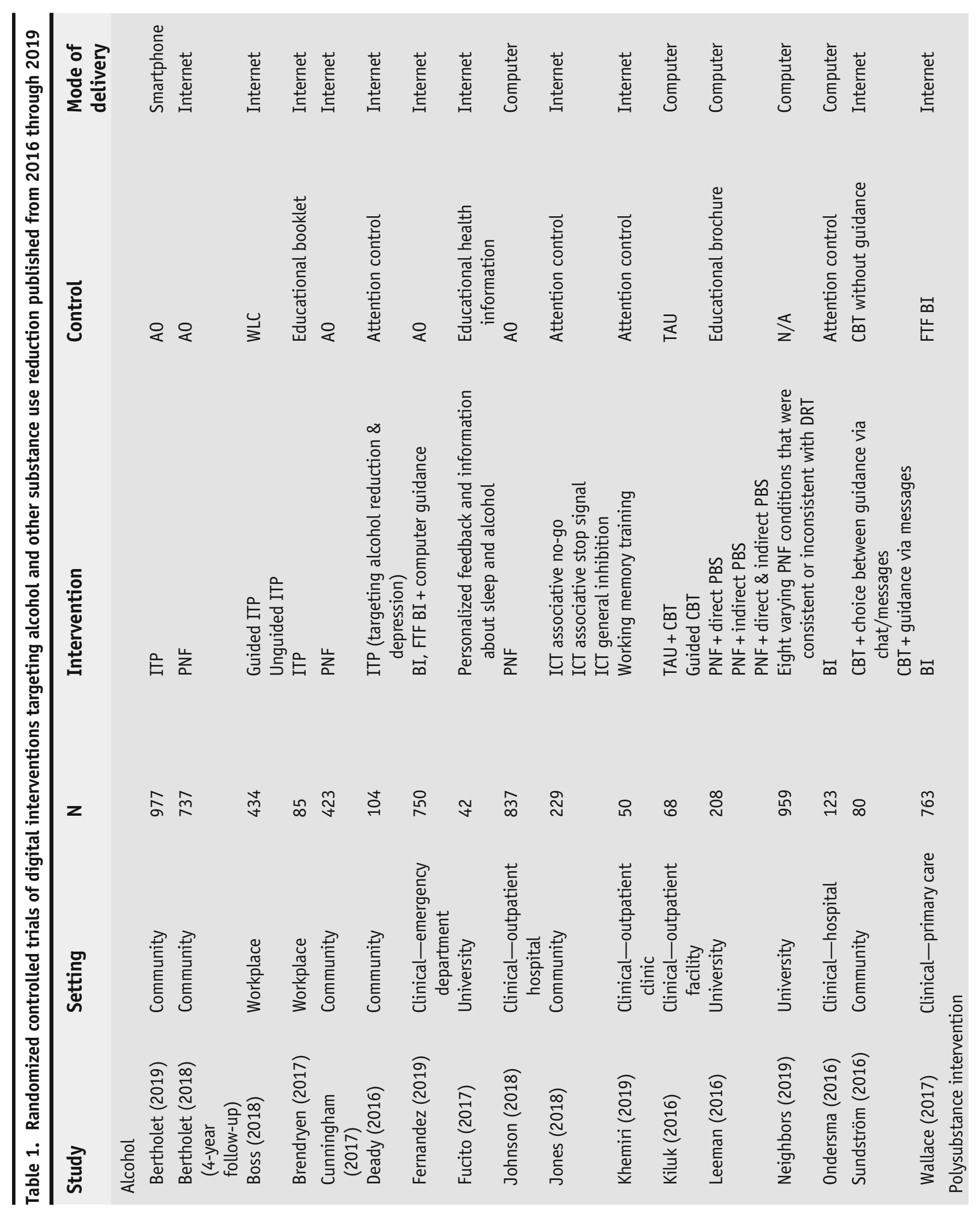




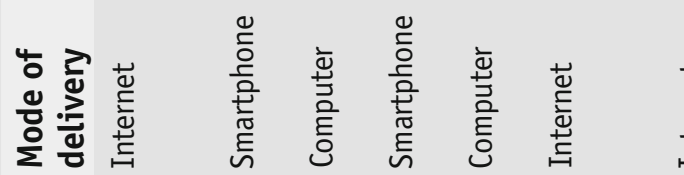

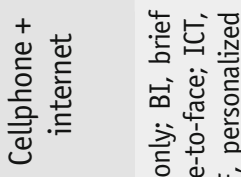

高岕范

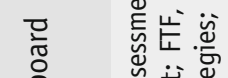

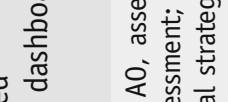

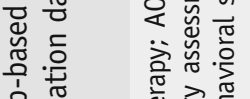

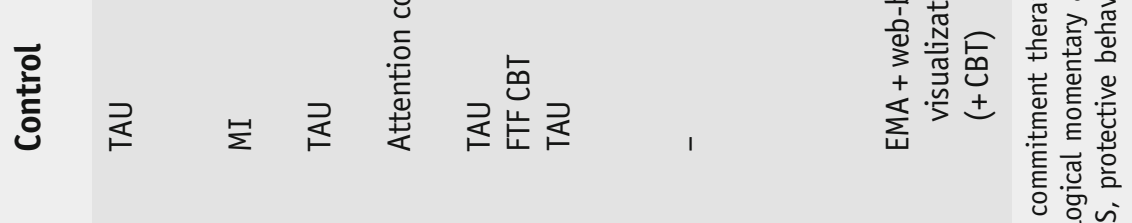

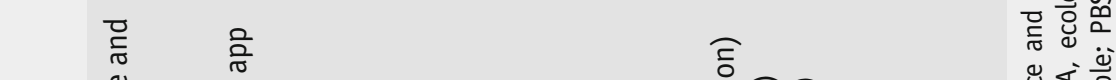

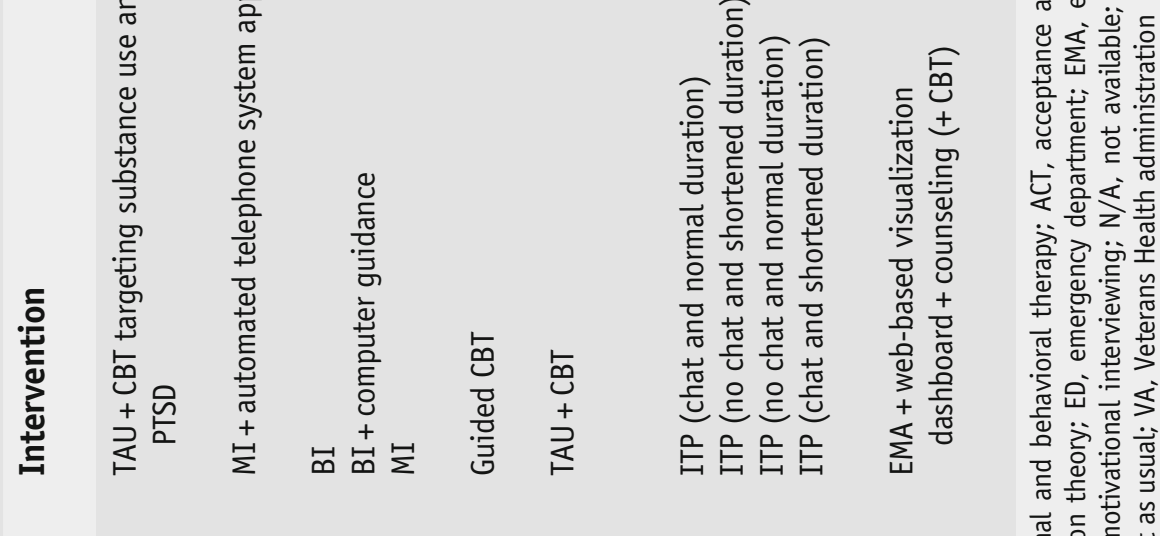

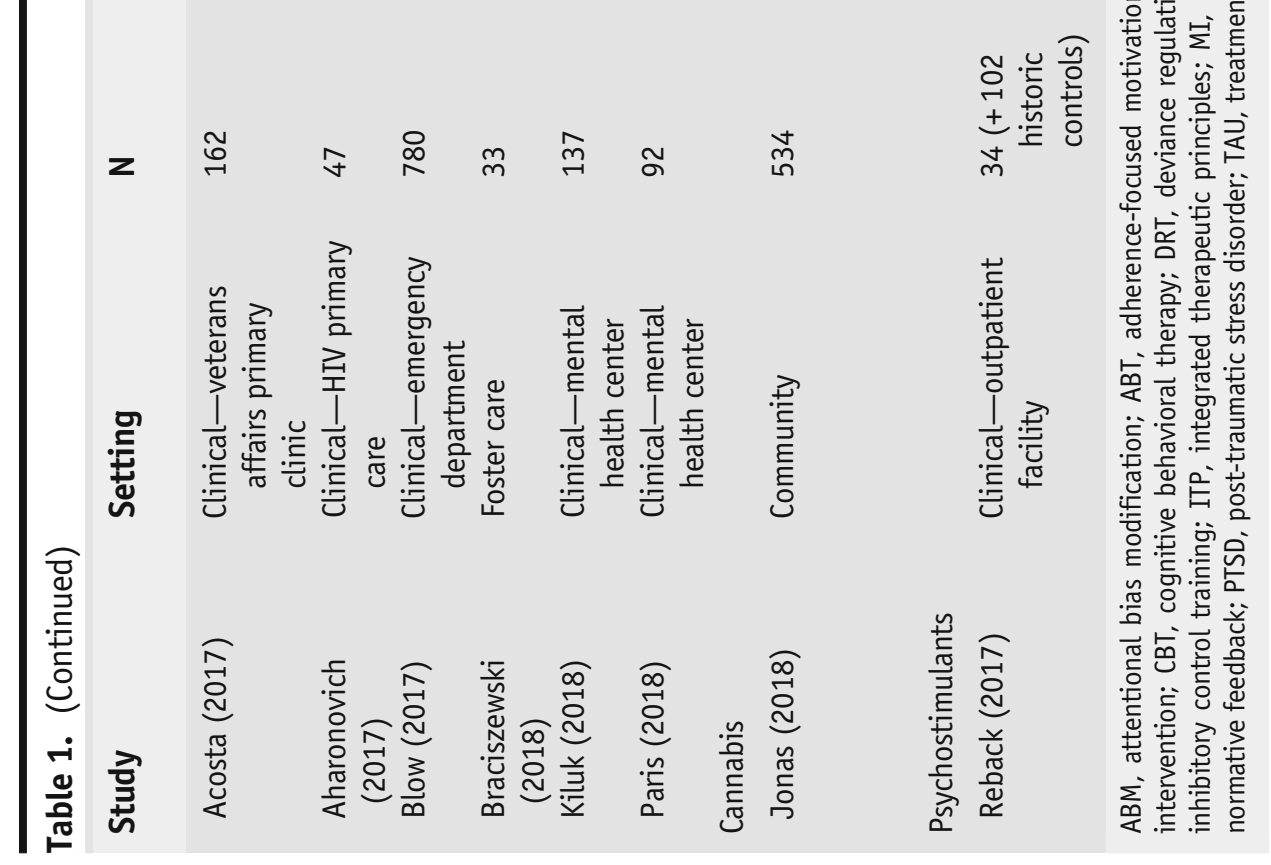


regarding individuals' substance use behavior compared with the perception they have of substance use norms within their specific reference group and with the actual substance use behavior in that particular group [21]. Two new RCTs applying digital PNF interventions and one 4-year follow-up PNF alcohol outcome study were identified. All identified studies investigated the effect of unguided PNF and compared them to assessment only (AO). Notably, a recent Individual Patient Data (IPD) meta-analysis [18•] concluded that PNF interventions are significantly less likely to decrease low-risk drinking compared to interventions using integrated therapeutic principles $(\mathrm{OR}=0.52)$.

PNF interventions are mostly used in young people, but less evidence is available for adults with more harmful drinking patterns. For this reason, the study by Johnson et al. [25] recruited 837 problem drinkers ( $>5$ and $<9$ on the Alcohol Use Disorders Identification Test [22] (AUDIT)) from a hospital outpatient setting and compared the PNF group with an AO condition. No significant between-group differences were found. Additionally, unguided internetbased PNF compared to an AO condition was also not found to be efficacious at four-year follow-up in a community sample of problem drinkers ( $>14$ drinks/ week, $\geq 6$ drinks/occasion at least monthly, or $\geq 8$ AUDIT score) [26], whereas a significant reduction in number of drinks per week had been shown during the 1-month follow-up. To our knowledge, this is the longest follow-up conducted for a PNF intervention. The results indicate that internet-based PNF interventions in community samples might lack long-term efficacy.

The study by Cunningham et al. [27] applied a novel recruitment strategy, using Mechanical Turk (MTurk) to recruit problem drinkers (AUDIT score $\geq 8$ ). MTurk is an online platform operated by Amazon offering registered individuals who are paid to perform certain tasks online (often surveys). Few psychological interventions have recruited participants via MTurk. The recruitment was extremely successful: 423 participants in $3.2 \mathrm{~h}$. Participants were randomized to internet-based PNF or AO. The 3-month follow-up rate was good (85\%); however, no significant between-group differences were found for the number of drinks in a typical week during follow-up. One disadvantage of paid online recruiting systems is that the samples are likely not representative of the target population and that the participants' primary goal is to be paid rather than receive the intervention. However, such novel online recruitment methods might be of value for testing new interventions or engaging problem drinkers in the co-creation process.

Brief interventions

Brief interventions (BIs) are concise interventions taking place over a brief period of time (typically 15-30 min) and are mostly applied in general practitioners' (GP) practices or hospital emergency departments (ED) [23]. BIs are solution-focused; they target patients' motivation and include personalized advice. Four published RCTs applying digital BIs have been published over the last three years.

A large number of studies assessing digital BIs have been conducted so far in community, hospital, and ED settings. However, given that a large number of problem drinkers have their first clinical contact through primary care, it is of great importance to evaluate the efficacy of digital Bis also in primary care settings. A review by Nair et al. [24] based on 15 small-scale studies hinted at the likely 
clinical and cost-effectiveness of digital interventions in primary care. However, no definite conclusions could be reached due to the small sample sizes of those studies. For this reason, Wallace et al. [25] recruited 763 adult problem drinkers ( $\geq 8$ on the AUDIT) from primary care during a visit to their GP and randomized them to unguided internet-based BI or to GP-delivered FTF BI. The unguided internet-based BI was significantly more effective (10\% reduction) in reducing the number of patients with an AUDIT score $\geq 8$, compared to the FTF BI condition (15\% increase). Nevertheless, because the authors identified bias in the final AUDIT question for their study design, specifically, asking whether patients received advice to reduce drinking from a healthcare professional, which likely elicited a positive response in the FTF BI condition, it is not possible to reach robust conclusions from this RCT. Further research is required to evaluate whether the findings can be replicated using more robust outcome measures.

Postpartum women are an important target group for BIs. Studies show that the majority of women reduce their drinking levels during pregnancy [26], but a substantial percentage return to pre-pregnancy levels after having given birth [27]. Few studies have been conducted applying digital BIs to reduce drinking during pregnancy $[28,29]$; however, postpartum drinking has received limited attention so far. Ondersma et al. [30] evaluated the effect of a computer-based BI on 7-day point-prevalence abstinence and drinking days among postpartum women. Postpartum women with an elevated risk for alcohol use $(n=123 ; \geq 4$ standard drinks at a time at least twice per month in the 12 months pre-pregnancy, and $\geq 2$ on the T-ACE [31]) were recruited and randomized to computer-based $\mathrm{BI}$ or AO. The results did not indicate significant group differences in decreased drinking levels among the participants. However, given the mixed findings in the broader BI literature regarding alcohol reduction in this target group, further research into the effective key elements of BIs should be conducted.

A meta-analysis by Schmidt et al. [32] has previously investigated the effects of BIs in ED settings on alcohol use reduction. They identified 28 studies of which four applied a digital intervention. They provided evidence that BIs conducted in EDs produce small statistically significant effects. The subgroup analysis including only digital interventions showed similar effect sizes for reduction in alcohol use; however, the effect was statistically non-significant. Given the inclusion of various comparators including TAU, leaflet only, specific interventions (unspecific, empathic advice), brief advice, and the fact that comparators, such as TAU, can vary substantially, the findings should be interpreted with caution.

Fernandez et al. [33] assessed potential non-inferiority of a computer-based BI compared to therapist-delivered BI in an ED setting. A total of 750 problem drinkers $(>3 />4$, women/men on AUDIT-C) were recruited among ED patients. The patients were randomized to (a) computer-based BI, (b) FTF BI, or (c) TAU consisting of a 3-5-min minimal information session with a staff member informing the patients about various health services. No significant between-group differences were found regarding overall alcohol use reduction across 12 months.

\section{Cognitive behavioral therapy}

With internet-based cognitive behavioral therapy (CBT), patients are exposed to similar components as in conventional CBT, by logging in regularly to an 
internet-based treatment platform to access, read, and download online materials to complete specific modules [34].

Two published RCTs applying digital CBT-based interventions for alcohol reduction have been identified. Digital CBT interventions have not been previously evaluated in a clinical population with alcohol use disorder (AUD). Kiluk et al. [35] recruited 68 treatment-seeking individuals with an AUD (according to DSM-IV criteria for alcohol abuse or dependence) from an outpatient addiction facility. Patients were randomized to (a) TAU-only or individual psychotherapy, (b) a computer-based intervention combined with TAU delivered in the outpatient facility, or (c) a computer-based intervention delivered in the outpatient facility in combination with brief weekly clinical monitoring. Computer-based CBT in combination with TAU produced a significantly greater percentage of abstinent days compared to TAU-only at post-treatment $(d=0.71)$. Additionally, clinical monitoring did not have an additional effect to computer-based CBT. Therefore, it appears that computer-based CBT delivered as add-on to TAU might be valuable for patients with AUD in outpatient addiction facilities. Future research should investigate the added value of digital CBT interventions when delivered outside of an outpatient facility (e.g., at the patient's home).

The number of studies that evaluated the effectiveness of therapist-delivered guided interventions are few, and even fewer studies have investigated the effectiveness of varying therapist-delivered guidance methods. Sundström et al. [36] recruited 80 treatment-seeking problem drinkers $(>6 />8$ AUDIT score for women/men) from the community. The participants were randomized into (a) an internet-based self-help program based on CBT with the choice between synchronous guidance via chat or asynchronous guidance via messages, (b) the CBT self-help program combined with asynchronous guidance via messages, and (c) the CBT self-help program with no guidance. The group receiving asynchronous guidance via messages showed reduced drinking at 10-week follow-up (10 standard glasses during past week) compared to the group which had the choice between synchronous or asynchronous guidance (11.6 standard glasses during past week). However, given the small sample size, this finding should be interpreted with caution. When the authors grouped both guidance groups together and compared their drinking levels against the no guidance condition, significantly lower past week alcohol consumption $(d=$ 0.77 ) was found. This result is in line with the IPD meta-analysis of Riper et al. $[18 \bullet]$ that indicated that guided interventions produce improved treatment outcomes compared to unguided interventions.

Integrated therapeutic principles

The term integrated therapeutic principles refers to interventions that make use of various elements from different psychological theories, such as CBT, MI, and PNF. Four studies using integrated therapeutic principles were identified.

Boss et al. [37] assessed the effectiveness of an internet-based intervention based on integrated therapeutic principles specifically targeting the German working population. Problem drinking employees $(n=434)$ (at least $14 / 21$ women/men standard units per week, and $\geq 6 / 8$, women/men on the AUDIT) were randomized to (a) internet-based $\mathrm{MI}+\mathrm{PNF}+$ emotion regulation with guidance, (b) unguided internet-based $\mathrm{MI}+\mathrm{PNF}+$ emotion regulation, or (c) waitlist control (WLC). The investigators found no significant difference 
between the guided and unguided conditions regarding weekly consumed standard drinks. However, the two experimental conditions were not sufficiently powered to detect statistically significant differences. In contrast, both of the experimental conditions were significantly more effective compared to WLC (4.9 standard drinks greater decrease on average). This study is one of the few indicating that internet-based alcohol interventions among risky drinkers from the working population can effectively reduce drinking levels.

The study by Brendryen et al. [38] sheds further light on digital interventions for employees. A total of 85 problem drinkers ( $\geq 3$ on the Fast Alcohol Screening Test (FAST) [39]) were randomized online via their workplace. After delivering PNF, the participants were allocated to an intensive unguided internetbased intervention or an educational alcohol information-only condition. The intensive intervention comprised 62 modules based on goal setting, relapse prevention, emotion regulation techniques, positive psychology, and CBT. At follow-up, there was no significant difference between the PNF + intensive intervention condition and the PNF + educational alcohol information-only condition regarding number of weekly drinks. Due to recruitment problems, the study was hampered by low statistical power, indicating that differences might have gone unnoticed. Future RCTs in occupational settings could benefit from the recruitment problems encountered in this study, such as postponed recruitment due to technical problems, overestimated recruitment potential due to earlier pilot testing within few enthusiastic companies, and unused employee email accounts. In addition, certain organizations declined participation due to inappropriate timing, random assignment of the participants to experimental or control conditions, or concerns about employees spending too much working time on the intervention.

Many smartphone applications to reduce unhealthy drinking are currently available; however, few have been assessed in terms of their effectiveness. Bertholet et al. [40] conducted one of the first RCTs and recruited 977 problem drinkers (AUDIT $\geq 8$ and drinking $\geq 15$ standard drinks per week) using the MTurk platform. Participants were randomized to receive AO or an unguided smartphone application. The application included several components, such as PNF, self-monitoring, and a blood alcohol content calculator. At 6-month follow-up, no significant differences were found between the conditions regarding number of drinks per week. However, download rates of the app were limited and individuals who downloaded the app appeared to benefit from it. Therefore, it may be concluded that simply providing access to a smartphone application is probably not sufficient to reduce drinking outcomes.

There is a high comorbidity of alcohol and depressive disorders [41]; however, only a limited number of studies focus on comorbid conditions while aiming for a reduction in drinking levels via a digital intervention. Deady et al. [42] targeted depressed, hazardous drinkers in the community. They recruited 104 young problem drinkers (AUDIT $\geq 8$ ) with moderate depression ( $\geq 7$ on the Depression Anxiety Stress Scale [43]) and randomized them to a transdiagnostic intervention consisting of internet-based CBT + MI intervention or a health information attention control condition. Results showed a significant reduction regarding number of drinks per week $(d=0.76)$ and depression symptom severity $(d=0.71)$ in the intervention group compared to the attention control condition. However, during the 6-month follow-up, the effects became non-significant. This study contributes valuable information to the 
digital transdiagnostic intervention field. However, given the observed low adherence and high attrition, further studies are required to better understand long-term outcomes.

\section{Reduction of cannabis use}

\section{Integrated therapeutic principles}

The number of RCTs applying a digital intervention targeting cannabis use is limited. A meta-analysis published in 2019 [19] has identified 20 RCTs targeting cannabis use, with the latest dating from 2016. Small but significant reductions in cannabis use were found for treatment interventions. In the last three years, only one RCT has been published. Jonas et al. [44] applied an internet-based intervention program that incorporated principles of a selfregulation and a self-control model of therapy for treatment of addictive behaviors and applied a solution-focused approach as well as MI. They investigated its efficacy by shortening the program or by varying the counseling between chat-based direct or time-lagged counseling, resulting in four conditions in a randomized factorial design. The results showed an overall reduction in cannabis use days $(d=2.05)$ and the number of use events $(d=1.21)$ in particular up to 12 months, but no significant differences between the four groups. However, the conditions receiving chat-based counseling reported higher treatment satisfaction $(d=0.34)$ and stronger working alliance $(d \geq$ 1.13) compared to conditions receiving time-lagged counseling. This indicates that the shortened and unguided version of the intervention was as effective as the full intervention combined with chat-based guidance. However, it might be preferable to include chat-based counseling to enhance patients' experience and thereby improve treatment adherence.

\section{Reduction of opioid use}

\section{Community reinforcement approach}

CRA is an extensive behavioral program supporting patients through functional analyses to investigate triggers and consequences of specific behaviors and to develop strategies to either avoid or address those behaviors. CRA makes use of various incentives of a vocational, social, and recreational nature in order to change patients' circumstances [45].

No RCTs applying a digital intervention targeting the reduction of opioid use have been published in the last three years. However, a meta-analysis from 2017 [20] has identified four RCTs [46-49], with the most recent from 2014, all of which applied a digital community reinforcement approach (CRA). From this meta-analysis, it appeared that CRA + TAU resulted in greater opioid use reductions compared to TAU-only $(g=0.36)$.

\section{Polysubstance interventions}

\section{Brief interventions}

The literature on polysubstance interventions is scarce. A meta-analysis [20] identified one RCT targeting multiple primary substances applying computerbased BI [50] compared to FTF BI reporting no effect of the intervention on substance use reduction. In the last three years, one RCT applying a digital 
guided polysubstance intervention based on BI has been conducted. Blow et al. [51] randomized 780 substance users at the hospital ED to (a) virtually guided BI performed on a tablet computer, (b) FTF BI with computer-supported guidance, or (c) TAU entailing educational information brochures on general health and HIV prevention. All interventions were provided at the ED. After three months, the participants were re-randomized to receive an adapted motivational enhancement therapy booster or a TAU booster. At 3-month followup, there were no differences in any drug use days for either BI condition compared to TAU, but there was an effect of FTF BI on the number of cannabis use days $(p=0.04)$. Averaged over 6 and 12 months, the FTF BI and TAU conditions showed a significant decrease in days using any drugs, whereas both BI conditions showed significantly fewer days of cannabis use compared to TAU. There were no additional effects of the booster on substance use.

\section{Motivational interviewing}

MI is a counseling approach developed by Miller and Rollnick [52]. With MI, the patient, rather than the therapist, is the one who voices the arguments for behavior change. In the last three years, two RCTs investigating digital MI-based polysubstance interventions have been conducted.

Braciszewski et al. [53] conducted one of the first studies to investigate the use of a variety of substances by youth exiting foster care $(n=33)$, and it is one of the few studies included in this review to include a long-term follow-up of twelve months. At every timepoint up to twelve months, the intervention group showed greater percentages of abstinent days from the drug of choice, with effect sizes ranging from $d=0.32$ to $d=0.62$. However, larger studies are warranted, in order to better generalize the effects, since the sample size in this study was too small to do so.

The pilot study of Aharonovich [54] is one of the few targeting HIV-positive, injecting drug users $(n=42)$ in primary care. A smartphone-based intervention containing self-monitoring, positive reinforcement, and personalized feedback as add-on to MI was tested. After two months, there was a greater reduction in the total number of drinking days and total number of drug use days compared to the control group receiving MI-only. Despite these positive results, RCTs with a greater sample size and long-term follow-ups are needed to better generalize the results and investigate long-term effects.

Three RCTs evaluating digital polysubstance CBT interventions have been identified. Kiluk et al. [55] recruited 137 treatment-seeking outpatients with an SUD in a mental health center and randomized them to guided computer-based CBT as add-on to weekly monitoring, FTF CBT, or TAU entailing weekly counseling. After 12 weeks, there was no difference between CBT groups, but both groups showed significantly greater reductions in substance use compared to TAU. Interestingly, for the guided computer-based CBT condition, these results were maintained at 6 months, but not for the FTF CBT condition. This might indicate that computer-based CBT in combination with weekly monitoring may be a valuable alternative to intensive therapist-delivered CBT, with possibly longer lasting results. However, given that the within-group sample sizes were relatively small, larger RCTs are needed to better generalize the effects. 


\section{Reduction of psychostimulant use}

Acosta et al. [56] recruited 162 problem drinking (AUDIT score $>8 />7$ for men/women) or substance misusing (DAST-10 score $\geq 2$ ) veterans via a Veterans Affairs (VA) primary clinic and randomized them to TAU, entailing usual VA primary care services, or to TAU in combination with unguided internetbased CBT, targeting symptoms of substance use and post-traumatic stress disorder (PTSD). The combined treatment produced significant reductions in heavy drinking but not for other substances, PTSD symptoms, or quality of life. Future studies should further investigate interventions to target comorbid psychiatric disorders in a greater sample.

Paris et al. [57] recruited 92 treatment-seeking individuals at an outpatient mental health center. They randomized the patients to either TAU consisting of weekly supportive counseling or TAU in combination with unguided internetbased CBT. At the end of the treatment, the internet-based + TAU condition showed significantly greater substance use reduction $(d=0.13)$.

\section{Ecological momentary assessment}

EMA is used to intensively assess behavioral experiences in a natural setting. Using a particular device (e.g., smartphone), the participants are prompted to report their experiences throughout the day [58].

One study augmented their 8-week gay-specific CBT intervention for gay and bisexual men, entailing male cultural references, to reduce methamphetamine use and risky sexual behavior in an outpatient methamphetamine abuse treatment program with ecological momentary assessment (EMA) for monitoring and counseling. Reback et al. [59] applied smartphone-based EMA for selfmonitoring to reduce methamphetamine use and risky sexual behavior, either with or without counseling. Augmenting CBT with EMA (with or without counseling) did not lead to greater amphetamine use reductions compared to CBT-only. In addition, counseling did not lead to significantly greater reduction in amphetamine use compared to no counseling.

\section{Conclusions}

Based on previous meta-analyses, one of the identified gaps was the need for more guided add-on, or blended interventions, compared to active control groups. The included studies from the last three years seem to more often investigate digital interventions as add-on compared to an active control group, but they still are mostly unguided. Seven studies investigated guided interventions, of which four focused on alcohol [33, 35-37], two on polysubstance interventions [51, 55], and one on psychostimulants [59]. Furthermore, the literature on psychostimulants and opioid interventions is still scarce, with only one study focusing on psychostimulant use, a guided add-on intervention compared to an active control group, and none focusing on opioids. However, given the increase in opioid use problems-opioid deaths in the US increased by $27.7 \%$ from 2015 to 2016 [60]—it would be desirable to focus on digital interventions for opioid use reduction.

A second gap was the variety of applied outcome measures when assessing substance use outcomes. To improve the consistency across RCTs, it is suggested 
that a set of standardized outcomes should be used, based on the relevant substance that is measured. Additionally, as most studies applied self-report measures, the use of toxicology screening should be considered as well, whenever feasible, to increase the reliability of substance use assessment.

Third, there is a need for longer follow-ups as most identified studies base their conclusions on post-treatment results or on 6-month follow-up. Since only a minority of studies included follow-up to 12 months and one study reported a 4-year follow-up, there is a scarcity of studies investigating long-term effects of digital interventions on substance use reduction.

Fourth, although some studies investigated polysubstance interventions, only two focused on interventions targeting substance use disorders with comorbid disorders, namely, depression and PTSD. Given the high prevalence of comorbid disorders in substance users [61], more attention should be devoted to those conditions.

The literature published over the last three years (2016-2019) shows advances with respect to targeting the gaps that existed before that period. Particular strengths of the literature include focusing on new target groups (e.g., patients with clinical AUD, young adults exiting foster care, or comorbid disorders), applying interventions in new settings and using novel recruitment strategies (e.g., Amazon's Mechanical Turk). Although future research should take into account the gaps that still remain, the current knowledge contributes to the development of more effective interventions to produce a substantial mental health impact.

\section{Compliance with Ethical Standards}

\section{Conflict of Interest}

Nikolaos Boumparis declares that he has no conflict of interest. Mieke H.J. Schulte declares that she has no conflict of interest. Heleen Ripper declares that she has no conflict of interest.

\section{Human and Animal Rights and Informed Consent}

This article does not contain any studies with human or animal subjects performed by any of the authors.

Open Access This article is distributed under the terms of the Creative Commons Attribution 4.0 International License (http://creativecommons.org/licenses/by/4.0/), which permits unrestricted use, distribution, and reproduction in any medium, provided you give appropriate credit to the original author(s) and the source, provide a link to the Creative Commons license, and indicate if changes were made.

\section{References and Recommended Reading}

Papers of particular interest, published recently, have been highlighted as: $\bullet$ Of importance

1. Global status report on alcohol and health 2018. Geneva: World Health Organization; 2018. Licence: CC BY-NC-SA 3.0 IGO.
2. United Nations Office on Drugs \& C World drug report 2018. Sales No. E.18.XI.9; 2018.

3. Degenhardt L, Charlson F, Ferrari A, Santomauro D, Erskine H, Mantilla-Herrara A, Whiteford H, Leung J, 
Naghavi M, Griswold M, Rehm J. The global burden of disease attributable to alcohol and drug use in 195 countries and territories, 1990-2016: a systematic analysis for the Global Burden of Disease Study 2016. The Lancet Psychiatry. 2018 Dec 1;5(12):987-1012.

4. Grant BF, Saha TD, Ruan WJ, Goldstein RB, Chou SP, Jung J, et al. Epidemiology of DSM-5 drug use disorder results from the national epidemiologic survey on alcohol and related conditions-III. JAMA Psychiatry. 2016;73:39-47.

5. Saha TD, Grant BF, Chou SP, Kerridge BT, Pickering RP, Ruan WJ. Concurrent use of alcohol with other drugs and DSM-5 alcohol use disorder comorbid with other drug use disorders:

sociodemographic characteristics, severity, and psychopathology. Drug Alcohol Depend.

2018; 187:261-9.

6. Yuodelis-Flores C, Ries RK. Addiction and suicide: a review. Am J Addict. 2015;24:98-104.

7. Hennessy EA, Fisher BW. A meta-analysis exploring the relationship between 12-step attendance and adolescent substance use relapse. J Groups Addict Recover. 2015;10:79-96.

8. Roozen HG, Boulogne JJ, Van Tulder MW, Van Den Brink W, De Jong CAJ, Kerkhof AJFM. A systematic review of the effectiveness of the community reinforcement approach in alcohol, cocaine and opioid addiction. Drug Alcohol Depend. 2004;74:1-13.

9. Magill M, Ray LA. Cognitive-behavioral treatment with adult alcohol and illicit drug users: a meta-analysis of randomized controlled trials. J Stud Alcohol Drugs. 2009;70:516-27.

10. Lussier JP, Heil SH, Mongeon JA, Badger GJ, Higgins ST. A meta-analysis of voucher-based reinforcement therapy for substance use disorders. Addiction. 2006;101:192-203.

11. Saloner B, Karthikeyan S. Changes in substance abuse treatment use among individuals with opioid use disorders in the United States, 2004-2013. JAMA.

2015;314:1515-7.

12. Schmidt LA. Recent developments in alcohol services research on access to care. Alcohol Res. 2016;38:27-33.

13. Lipari RN, Park-Lee E, Van Horn S. America's need for and receipt of substance use treatment in 2015. In The CBHSQ Report 2016 Sep 29. Substance Abuse and Mental Health Services Administration (US).

14. Behavioral Health Statistics and Quality (CBHSQ). Results from the 2017 national survey on drug use and health: detailed tables. Rockville; 2018.

15. Xu J, Rapp RC, Wang J, Carlson RG. The multidimensional structure of external barriers to substance abuse treatment and its invariance across gender, ethnicity, and age. Subst Abus. 2008;29:43-54.

16. Substance Abuse and Mental Health Services, Administration. Key substance use and mental health indicators in the United States: results from the 2017 national survey on drug use and health. Rockville: Center for Behavioral Health Statistics and Quality, Substance Abuse and Mental Health Services Administration; 2018.
17. Taylor CB, Luce KH. Computer- and internet-based psychotherapy interventions. Curr Dir Psychol Sci. 2003;12:18-22.

18. Riper H, Hoogendoorn A, Cuijpers P, Karyotaki E, Boumparis N, Mira A, Andersson G, Berman AH, Bertholet N, Bischof G, Blankers M. Effectiveness and treatment moderators of internet interventions for adult problem drinking: An individual patient data meta-analysis of 19 randomised controlled trials. PLoS medicine. 2018 Dec 18;15(12):e1002714.

Unique IPD meta-analysis about the effectiveness and treatment moderators of digital interventions for adult problem drinking.

19. Boumparis N, Loheide-Niesmann L, Blankers M, Ebert DD, Korf D, Schaub MP, et al. Short- and long-term effects of digital prevention and treatment interventions for cannabis use reduction: a systematic review and meta-analysis. Drug Alcohol Depend. 2019;200:82-94.

Recent systematic review and meta-analysis about the effectiveness of digital interventions for the reduction of cannabis use.

20. Boumparis N, Karyotaki E, Schaub MP, Cuijpers P, Riper H. Internet interventions for adult illicit substance users: a meta-analysis. Addiction. 2017. https:// doi.org/10.1111/add.13819.

Recent meta-analysis about the effectiveness of digital interventions for adult illicit substance use.

21. Dotson KB, Dunn ME, Bowers CA. Stand-alone personalized normative feedback for college student drinkers: a meta-analytic review, 2004 to 2014. PLoS One. 2015;10:e0139518.

22. Allen JP, Litten RZ, Fertig JB, Babor T. A review of research on the alcohol use disorders identification test (AUDIT). Alcohol Clin Exp Res. 1997;21:613-9.

23. Moyer A, Finney JW. Brief interventions for alcohol misuse. CMAJ. 2015;187:502-6.

24. Nair NK, Newton NC, Shakeshaft A, Wallace P, Teesson $\mathrm{M}$. A systematic review of digital and computer-based alcohol intervention programs in primary care. Curr Drug Abuse Rev. 2015;8:111-8.

25. Wallace P, Struzzo P, Della Vedova R, Scafuri F, Tersar $\mathrm{C}$, Lygidakis $\mathrm{C}$, et al. Randomised controlled noninferiority trial of primary care-based facilitated access to an alcohol reduction website. BMJ Open. 2017;7:e014576.

26. Floyd RL, Ebrahim S, Tsai J, O'Connor M, Sokol R. Strategies to reduce alcohol-exposed pregnancies. In: Matern. Child Health J. US: Springer; 2013. p. 1735-5.

27. Office of Applied Studies Substance use among women during pregnancy and following childbirth 2009.

28. Tzilos GK, Sokol RJ, Ondersma SJ. A randomized phase I trial of a brief computer-delivered intervention for alcohol use during pregnancy. J Women's Health. 2011;20:1517-24.

29. Ondersma SJ, Beatty JR, Svikis DS, Strickler RC, Tzilos GK, Chang G, et al. Computer-delivered screening and brief intervention for alcohol use in pregnancy: a pilot 
randomized trial. Alcohol Clin Exp Res.

2015;39:1219-26.

30. Ondersma SJ, Svikis DS, Thacker LR, Beatty JR, Lockhart N. A randomised trial of a computer-delivered screening and brief intervention for postpartum alcohol use. Drug Alcohol Rev. 2016;35:710-8.

31. Sokol RJ, Martier SS, Ager JW. The T-ACE questions: practical prenatal detection of risk-drinking. Am J Obstet Gynecol. 1989;160:863-8 discussion 868-70.

32. Schmidt CS, Schulte B, Seo H-N, Kuhn S, O'Donnell A, Kriston $\mathrm{L}$, et al. Meta-analysis on the effectiveness of alcohol screening with brief interventions for patients in emergency care settings. Addiction. 2016;111:783-94.

33. Fernandez AC, Waller R, Walton MA, Bonar EE, Ignacio RV, Chermack ST, et al. Alcohol use severity and age moderate the effects of brief interventions in an emergency department randomized controlled trial. Drug Alcohol Depend. 2019;194:386-94.

34. Andersson G, Titov N. Advantages and limitations of internet-based interventions for common mental disorders. World Psychiatry. 2014;13:4-11. https://doi. org/10.1002/wps.20083.

35. Kiluk BD, Devore KA, Buck MB, Nich C, Frankforter TL, LaPaglia DM, et al. Randomized trial of computerized cognitive behavioral therapy for alcohol use disorders: efficacy as a virtual stand-alone and treatment add-on compared with standard outpatient treatment. Alcohol Clin Exp Res. 2016;40:1991-2000.

36. Sundström C, Gajecki $M$, Johansson $M$, Blankers $M$, Sinadinovic K, Stenlund-Gens E, et al. Guided and unguided internet-based treatment for problematic alcohol use - a randomized controlled pilot trial. PLoS One. 2016;11:e0157817.

37. Boß L, Lehr D, Schaub MP, Paz Castro R, Riper H, Berking $M$, et al. Efficacy of a web-based intervention with and without guidance for employees with risky drinking: results of a three-arm randomized controlled trial. Addiction. 2018;113:635-46.

38. Brendryen $\mathrm{H}$, Johansen A, Duckert F, Nesvåg S. A pilot randomized controlled trial of an internet-based alcohol intervention in a workplace setting. Int J Behav Med. 2017;24:768-77.

39. Hodgson R, Alwyn T, John B, Thom B, Smith A. The FAST alcohol screening test. Alcohol Alcohol. 2002;37:61-6.

40. Bertholet N, Godinho A, Cunningham JA. Smartphone application for unhealthy alcohol use: pilot randomized controlled trial in the general population. Drug Alcohol Depend. 2019;195:101-5.

41. Riper H, Andersson G, Hunter SB, de Wit J, Berking M, Cuijpers $P$. Treatment of comorbid alcohol use disorders and depression with cognitive-behavioural therapy and motivational interviewing: a meta-analysis. Addiction. 2014;109:394-406.

42. Deady M, Mills KL, Teesson M, Kay-Lambkin F. An online intervention for co-occurring depression and problematic alcohol use in young people: primary outcomes from a randomized controlled trial. J Med Internet Res. 2016;18:e71.
43. Brown TA, Chorpita BF, Korotitsch W, Barlow DH. Psychometric properties of the depression anxiety stress scales (DASS) in clinical samples. Behav Res Ther. 1997;35:79-89.

44. Jonas B, Tensil M-D, Tossmann P, Strüber E. Effects of treatment length and chat-based counseling in a webbased intervention for Cannabis users: randomized factorial trial. J Med Internet Res. 2018;20:e166.

45. Meyers RJ, Villanueva M, Smith JE. The community reinforcement approach: history and new directions. J Cogn Psychother. 2005;19:247-60.

46. Bickel WK, Marsch LA, Buchhalter AR, Badger GJ. Computerized behavior therapy for opioid-dependent outpatients: a randomised control trial. Exp Clin Psychopharmacol. 2008;16:132-43.

47. Christensen DR, Landes RD, Jackson L, Marsch LA, Mancino MJ, Chopra MP, et al. Adding an internetdelivered treatment to an efficacious treatment package for opioid dependence. J Consult Clin Psychol. 2014;82:964-72.

48. Chopra MP, Landes RD, Gatchalian KM, Jackson LC, Buchhalter AR, Stitzer ML, et al. Buprenorphine medication versus voucher contingencies in promoting abstinence from opioids and cocaine. Exp Clin Psychopharmacol. 2009; 17:226-36.

49. Marsch LA, Guarino H, Acosta M, et al. Web-based behavioral treatment for substance use disorders as a partial replacement of standard methadone maintenance treatment. J Subst Abus Treat. 2014;46:1-26.

50. Schwartz RP, Gryczynski J, Mitchell SG, Gonzales A, Moseley A, Peterson TR, et al. Computerized versus inperson brief intervention for drug misuse: a randomized clinical trial. Addiction. 2014;109:1091-8.

51. Blow FC, Walton MA, Bohnert ASB, Ignacio RV, Chermack S, Cunningham RM, et al. A randomized controlled trial of brief interventions to reduce drug use among adults in a low-income urban emergency department: the Healthi ER You study. Addiction. 2017;112:1395-405.

52. Rollnick S, Miller WR. What is motivational interviewing? Behav Cogn Psychother. 1995;23:325-34.

53. Braciszewski JM, Tzilos Wernette GK, Moore RS, Bock BC, Stout RL, Chamberlain P. A pilot randomized controlled trial of a technology-based substance use intervention for youth exiting foster care. Child Youth Serv Rev. 2018;94:466-76.

54. Aharonovich E, Stohl M, Cannizzaro D, Hasin D. HealthCall delivered via smartphone to reduce cooccurring drug and alcohol use in HIV-infected adults: a randomized pilot trial. J Subst Abus Treat. 2017;83:15-26.

55. Kiluk BD, Nich C, Buck MB, Devore KA, Frankforter TL, LaPaglia DM, et al. Randomized clinical trial of computerized and clinician-delivered CBT in comparison with standard outpatient treatment for substance use disorders: primary within-treatment and follow-up outcomes. Am J Psychiatry. 2018;175:853-63.

56. Acosta MC, Possemato K, Maisto SA, Marsch LA, Barrie $\mathrm{K}$, Lantinga L, et al. Web-delivered CBT reduces heavy 
drinking in OEF-OIF veterans in primary care with symptomatic substance use and PTSD. Behav Ther. 2017;48:262-76.

57. Paris M, Silva M, Añez-Nava L, Jaramillo Y, Kiluk BD, Gordon MA, et al. Culturally adapted, web-based cognitive behavioral therapy for Spanish-speaking individuals with substance use disorders: a randomized clinical trial. Am J Public Health. 2018;108:1535-42.

58. Shiffman S, Stone AA, Hufford MR. Ecological momentary assessment. Annu Rev Clin Psychol. 2008;4:1-32.

59. Reback CJ, Rünger D, Fletcher JB, Swendeman D. Ecological momentary assessments for self-monitoring and counseling to optimize methamphetamine treatment and sexual risk reduction outcomes among gay and bisexual men. J Subst Abus Treat. 2018;92:17-26

60. Vivolo-Kantor AM, Seth P, Gladden RM, Mattson CL, Baldwin GT, Kite-Powell A, et al. Vital signs: trends in emergency department visits for suspected opioid overdoses-United States, July 2016-September 2017. MMWR Morb Mortal Wkly Rep. 2018. https://doi.org/ 10.15585/mmwr.mm6709e1.

61. Lai HMX, Cleary M, Sitharthan T, Hunt GE. Prevalence of comorbid substance use, anxiety and mood disorders in epidemiological surveys, 1990-2014: a systematic review and meta-analysis. Drug Alcohol Depend. 2015;154:1-13.

\section{Publisher's Note}

Springer Nature remains neutral with regard to jurisdictional claims in published maps and institutional affiliations. 